\title{
On High Frequency Polar Motion and Length of Day Variations
}

\author{
K. Arfa-Kaboodvand, E. Groten \\ Darmstadt University of Technology, Institute for Physical Geodesy, \\ Darmstadt, Germany
}

\begin{abstract}
The 0.042-day Earth rotation data (diurnal and semidiurnal) computed by the International GPS Service were used to analyze the daily/sub-daily variations of polar motion (PM) and length of day (LOD). Systematic and advanced spectral analytical investigations of the degree of periodic variability have been carried out. They show that the prominent periodical components can be found at the tidal frequencies of zonal, tesseral and sectorial waves. These investigations should give better insight into the physical processes, which influence Earth orientation (i.e. due to the atmospheric and oceanic motions, tidal forces etc.). It should be the basis for the detailed modeling of excitation functions in the sub-diurnal range of the high-frequency spectrum.
\end{abstract}

\section{Introduction}

Frank Stacy once compared polar motion research with a "never ending story," because with each solved problem two new and recent questions would come up, which need additional investigation. With new measurements of increased precision and accuracy we could attack completely new areas of polar motion research, such as the sub-diurnal range of the spectrum. In this area the interaction between the tidal and non-tidal effects and the combination of atmospheric and oceanic influences can become relevant. With recent GPS and VLBI data a mixture of densely (GPS) and sparsely (VLBI) distributed data are available, as far as the regional distribution is concerned. In time the observations may be separated by only one hour or so. We are well able to investigate periods shorter than terdiurnal effects.

The combined effect of limited resolution in tidal bands together with observational errors makes the complete separation of tidal from non-tidal constituents in Universal Time (UT1) and polar motion observations problematic. In general, we know very much about excitations with periods up to 65 weeks in UT1 and polar motion. There are severe gaps in the remaining part of the spectrum, where the role of the atmosphere (mainly in terms of atmospheric angular momentum (AAM) variations) is dominant in those medium-frequencies. In a variety of special studies we tried to get insight into the variable periods of sub-diurnal polar motion, and UT1 or length of day (LOD), derived from GPS observations. To what extent the tidal effects in GPS orbits are fully eliminated is not absolutely clear, so the oceanic and Earth tidal perturbation may some- 
how affect some of our results. Such tidal remnant influence may be negligible, if it exists at all.

Contrary to the past, there are now more detailed information and many data are available for oceanic and atmospheric variability. This makes the correlation and regression studies feasible, which should be the basis for the detailed modeling of excitation functions.

Most investigations in the field of high-frequency variations of the Earth rotation are mainly focused on the diurnal and semidiurnal variations in the Earths rotation rate (e.g. Freedman et al. 1994, Ray et al. 1994, Lichten et al. 1992 etc.). Therefore, the analysis and modeling of variations in Earth rotation at high-frequencies (periods shorter than or equal to one day) domain, especially the polar motion, is the main goal of our investigations. These variations are predominantly the result of the changes in the oceanic height and current due to the tidal potential generated by the Sun and Moon. Global numerical models of ocean tides may be used to predict variations in Earth rotation, i.e. Brosche et al. (1989) or Seiler (1991), but also some atmospheric excitations are to be expected (Freedman, 1994).

\section{Earth rotation data}

The almost 3 years of Earth rotation data used in this work stems from the Center for Orbit Determination (CODE). It was computed from the global IGS network (include 40 to 90 sites) and has a sampling interval of approximately 0.042 days. To remove the low-frequency variations (e.g. annual parts) in the polar motion, a smooth a priori polar motion series, e.g. Bulletin A series from the International Earth Rotation Service (IERS) was used. The IERS set was subtracted from the CODE after a spline interpolation (Figure 1) to cover the data gaps in the Bulletin A. We used also other classical and improved interpolation methods, e.g. least-squares adjustment (LSA) or generalized spline method (GSM). However they cannot serve as stand-alone methods to eliminate periods above one day. To obtain diurnal and sub-diurnal components after LSA or GSM, two different methods were applied: an enhanced Butterworth high-pass filtering (BHF) and a trigonometric interpolation (TI). More detailed information about these methods can be found in a paper by Arfa-Kaboodvand et al. (in preparation). Since our interest focuses on the daily and sub-daily polar motion, the first method seems to be the best solution of the present study. Additionally, it could be the basis for further modeling and model improvement (e.g. Rothacher 1998). For LOD we applied the LSA in combination with the trigonometric interpolation. In case of TI the LOD constitute a stochastic process with a zero mean value. The interpolant is, in this case, a cosine function with amplitude and phase as unknowns. The period is a positive integer value. The amplitude and phase of TI could be estimated by means of least-squares method from the LOD data. This procedure allows us to remove components of a specific period.

It should be pointed out that the simultaneous estimation of the sub-daily polar motion and orbital elements of the GPS satellite leads, due to their correlation, to huge retrograde diurnal terms. Therefore any exactly diurnal retrograde signal was suppressed by using special a priori constraints (Rothacher 

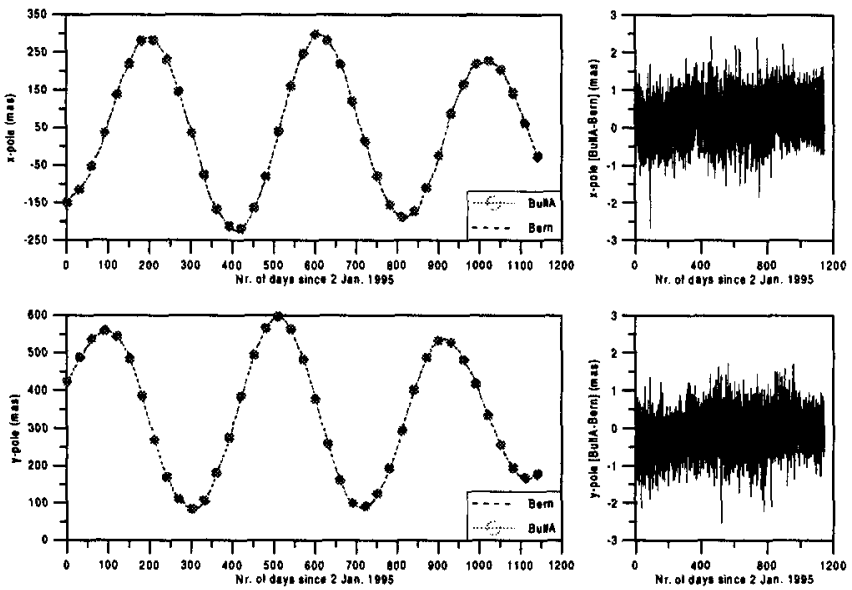

Figure 1. The upper and lower diagrams at the left side show the $\mathrm{x}$ and y-pole data from the Astronomical Institute of University of Berne (dash line) and from IERS (Bulletin-A series). Both diagrams at the right side show the differences in $\mathrm{x}$ - and $\mathrm{y}$-pole values among the 2 sets.

1998). However, our analysis indicated some diurnal retrograde signals within the 3 years of data. The 3 years of Earth orientation data consists of 5 different subsets, considering different kind of orbital models (classical or extended) or observational numbers and so on, so maybe these are responsible for that. Readers interested in more detailed information related to the preparation of Earth rotation data are referred to Rothacher (1998).

\section{Numerical methods and results}

The discrete deterministic modelling of various parts of the short-period spectrum in Earth rotation data did not yet lead to conclusive results and spurious systematic variations in Earth rotation data, such as seasonal effects obviously do exist (Arfa-Kaboodvand et al. 1999), so a systematic search on the degree of variability of periods including tidal (diurnal etc.) frequencies appeared useful. The basic problem of such studies lies in the imperfection of eliminating direct tidal effects, which induce in the various bands apparent variability of periods. That simply reflects imperfection of the tidal model containing nearly tidal frequencies that cannot be exactly taken into account in the data analysis. On the other hand, in the sub-diurnal frequency range, mainly oceanic influences could be identified by detecting pronounced frequency modulation or nonlinearities.

In the prediction of high frequency polar motion two-dimensional approaches are basically performed. They better take account of correlation between $\mathrm{x}$ - and $y$-components reflecting the physical meaning of the motion of the Earth's rotation axis. In view of interruptions of satellite orbit data we consequently used 

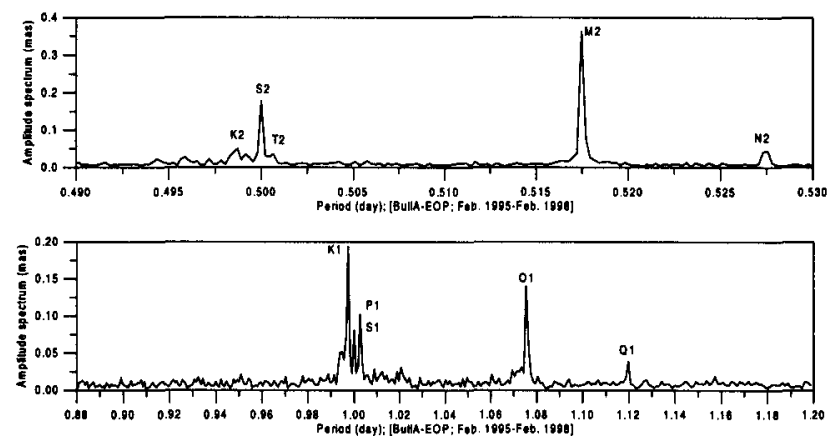

Figure 2. Amplitude spectra of the diurnal and semidiurnal tidal frequency bands generated from the 1140 days of polar motion data.

different spectral analysis techniques (e.g. Fourier method, Maximum Entropy) as reference (with fixed periods) in the non-tidal and tidal bands of the subdiurnal ranges and compared the results with those obtained from the complex Wavelet-analyses (Arfa-Kaboodvand et al., 1999). Appendix A gives a first impression of the important Wavelet formulas.

It should be emphasized that in the complex wavelet techniques used here, the polar motion has to be in the form $\vec{p}(t)=x_{p}(t)-j y_{p}(t)$, where $j=\sqrt{-1}$. Any other approach will lead to wrong energy density values, e.g. in retrograde semidiurnal range, if we do not change the Wavelet definition (Appendix A).

Note that by convention the positive direction is defined to be along the meridian at $90^{\circ} \mathrm{W}$ longitude, whereas the positive $\chi_{2}$ direction $\left(\chi_{1}\right.$ and $\chi_{2}$ are the equatorial components and are associated with the excitation of polar motion, according to Barnes et al. 1983) is taken to be along the meridian at $90^{\circ}$ E longitude, thus the minus sign in the above definition of $\vec{p}(t)$ corresponds with the theory of excitation. Note that the energy density results would be the same if we reverse the sign of angular frequency in the Wavelet transform. In this case we can use the polar motion in the original form $\vec{p}(t)=x_{p}(t)+j y_{p}(t)$.

Figure 2 shows the amplitude spectra for the short periodic, diurnal and semidiurnal frequencies. Similar analyses have been done also for the LOD. The biggest anomaly in the semidiurnal frequency band corresponds with the main lunar wave $M_{2}$. We notice that the $S_{1}$ and $S_{2}$ constituents are larger than those given by the theory of terrestrial and oceanic tides. This phenomenon may be due more to meteorology than atmospheric tides (Zharov et al., 1996).

Peter Varga (private communication) came recently to the following conclusion: "The polar motion anomalies of diurnal frequency band are probably of the Earth tidal origin and the oceanic tidal influence can generate semidiurnal LOD components."

Figure 3 shows the maximum energy density distribution of diurnal and semidiurnal polar motion as a function of time (III, IV) and period (I, II). Similar analysis has been done for the retrograde case of polar motion. As it was mentioned before, any exactly diurnal retrograde signal has been suppressed 

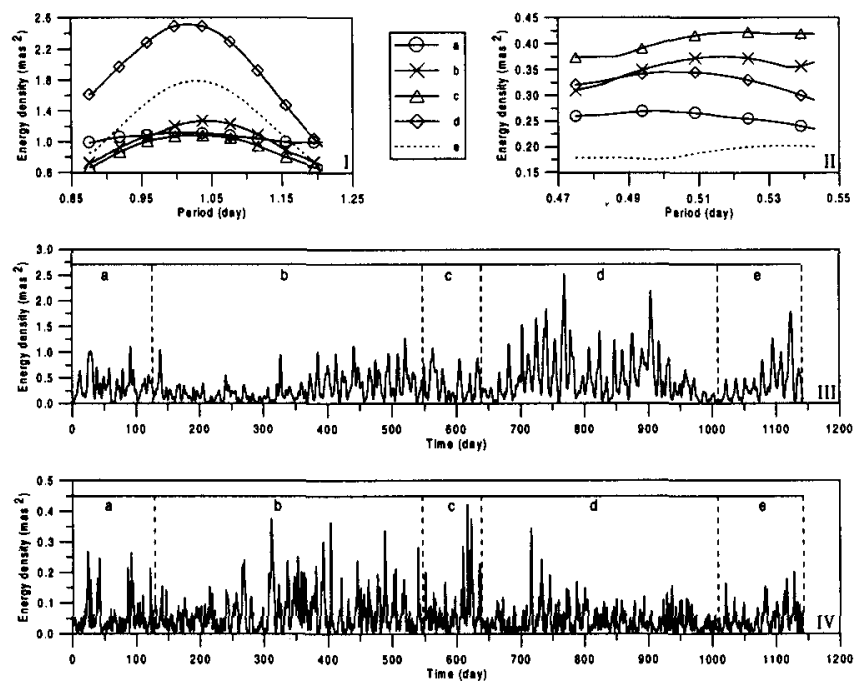

Figure 3. Maximum Energy density spectra of the diurnal and semidiurnal prograde polar motion generated from the 1140 days of polar motion data. Plots I and III show the daily maximum energy density and their corresponding periods. Plots II and IV show the sub-daily maximum energy density and their corresponding periods. The dotted vertical lines in III and IV mark the start and end of the different parts of the series (series a to e), as described in Rothacher (1998). 

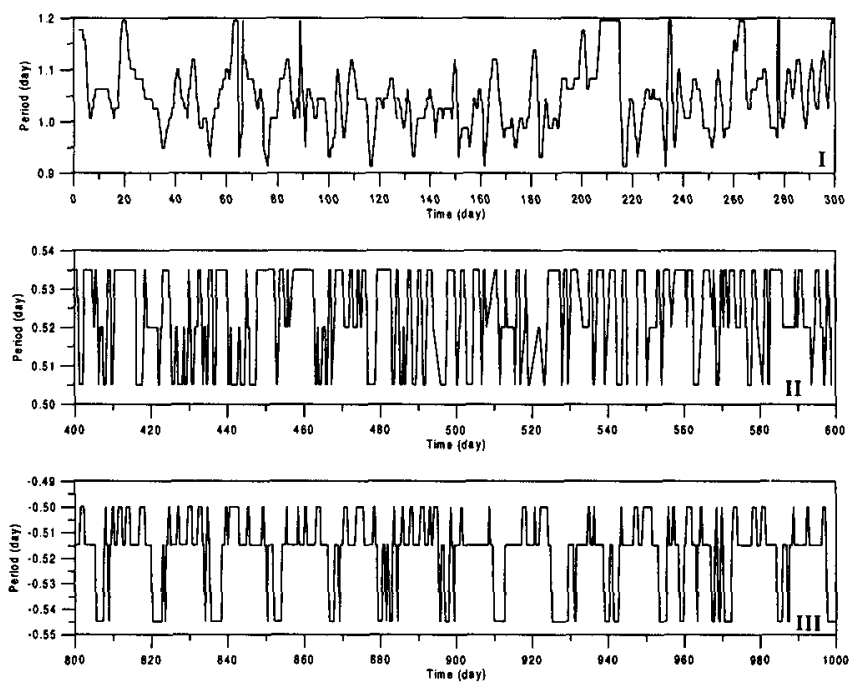

Figure 4. Period variation of the diurnal/semidiurnal prograde and semidiurnal retrograde polar motion generated from the 1140 days of data. Plot I shows the daily period variation of the prograde polar motion, and plots II and III show the pro- and retrograde period variations. All period variation lines refer to the maximum energy density (see Fig. 3). Note that the time axes are drawn in different time intervals to show the period variation within the whole data set.

in the estimation of polar motion. Nevertheless we found some peaks in the diurnal retrograde energy density, especially in the middle of the data series $d$ and at the end of series $b$. However, compared to the prograde semidiurnal case, which covers the lower range of the maximum diurnal prograde and semidiurnal retrograde energy density, the density values were not significant. Both upper diagrams in Figure 3 ( $\mathrm{I}$ and II) show that the diurnal prograde polar motion is more energetic (e.g. series $\mathrm{d}$ and e) than the semidiurnal prograde. The maximum energy density range of semidiurnal retrograde polar motion is a little smaller in magnitude than the diurnal progrades, but they are distributed more evenly, also compared to the diurnal retrograde.

We also notice that the period variation in diurnal and semidiurnal prograde case is stronger than in semidiurnal retrograde case. It is well-known that tides play a dominant roll in exciting sub-daily polar motion. Nearly $60 \%$ of the subdaily polar motion and approximately $90 \%$ of the corresponding UT1 power can be explained by the tidal constituents (Chao et al. 1996). Therefore the model of tidal variations in polar motion is important. Considering the Liouville equation (Munk and McDonald, 1960) the complex polar motion can be expressed as a linear combination of exponential functions. 


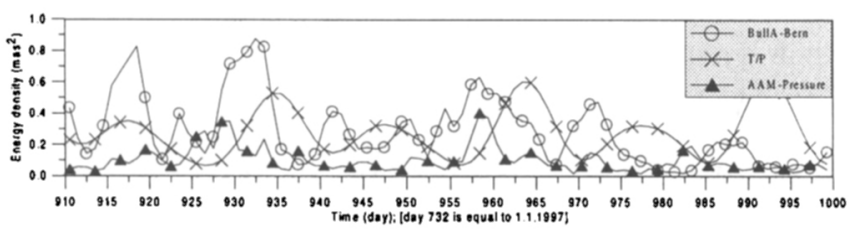

Figure 5. Energy density distribution of prograde polar motion (BulletinA-Bern), tidal variations (TOPEX/Poseidon model C) and AAM-pressure (reanalyzed NMC).

We notice a drift from $\mathrm{T} / \mathrm{P}$ relative to the polar motion data. This drift is probably due to sysematic effect in the GPS data such as orbit mismodeling in the GPS estimation process. So for further investigations this drift should be removed (see also Rothacher: preparation of sub-daily EOP).

\section{Conclusion}

The major parts of the studies in the field of Earth rotation focus on the variation of the Earth's rotation rate. The kinematics and dynamic complications of polar motion set it apart from the LOD investigation. However, we try to get more insight into both polar motion and length of day. We have to be fully aware that modeling errors inherent in solid and fluid Earth tidal effects may perturb interpretations of tidal as well as non-tidal components. Consequently, not all frequency modulations may be real.

Because of the conciseness and short length of the Earth rotation data, it is not possible to realize any significant yearly coherence between the prograde and retrograde spectra, or LOD. However, we also discovered some correlations between the prograde and retrograde spectra in polar motion. Since the daily/sub-daily variability due to AAM is quite small compared to oceanic tidal effects, further research is necessary to model, investigate and reduce the Earth rotation data. The motion of the rotation axis around the conventional terrestrial reference axis of the Earth, reflecting the physical motion of the deformable Earth in the "space-fixed" system related to the Ephemeris Pole, should reveal global deformations of the fluid and solid parts of the Earth. Similar effects in the long-period part were found, e.g., for El Niño ocean deformation and associated Earth rotation perturbations. The precisely defined tidal band makes it attractive to look for similar and also particular aspects in the sub-diurnal spectral areas, particularly as we learn more about frequency modulation in dynamic oceanic short-period motion. Detailed data analysis leads to seasonal and other systematic effects which do exist. It is necessary to study additional longer data series before stochastic studies, as described here, can lead to systematic deterministic modeling. However, deterministic modeling of significant excitations (for predicting short-term Earth rotation parameters over longer intervals) is still difficult. 
Acknowledgments. The support of Deutsche Forschungsgemeinschaft is appreciated. The data were kindly provided by the Astronomical Institute of the University of Bern.

\section{Appendix A}

The complex Wavelet technique is one of the significant features that makes it interesting for analyzing the Earth's rotation. The Wavelet method inhabits a representation of function space that is neither in the spectral, nor in the temporal, domain, but rather somewhere in-between. Consolidated information relating the ulterior motivations for using the Wavelet transform to analyze Earth rotation data and some detailed theoretical background of Wavelet and its expansion as a function of three parameters (a) dilation or compression, and (b) shifting and (c) stretching or subsiding factor has been presented in a paper by Arfa-Kaboodvand and Groten (1998).

In this section the authors describe briefly the essential definitions and extensions of the Wavelet technique that are significant for analyzing polar motion data. The main advantage of the Wavelet technique lies in the simultaneous time and frequency localization. The signal (here polar motion data) will be scanned through a basis function (so-called mother wavelet) in the time and frequency domains. The correct choice of the basis Wavelet function (Morlet, Mexican hat etc.) is decisive for the process development. Popiński and Kosek (1994) recommended for Earth rotation analysis the Morlet-Wavelet with a constant angular frequency of $\omega_{0}=\pi(2 / \ln 2)^{0.5}$. We start with the Morlet Wavelet function:

$$
\psi_{M}(t)=e^{j \omega_{0} t}\left(e^{-0.5 t^{2}}-\sqrt{2} e^{-0.25 \omega_{0}^{2}} e^{-t^{2}} \text { with } j=\sqrt{-1}\right.
$$

To improve the sidelobe behavior of the Morlet-function and reach a better frequency separation, we introduce a new parameter $\xi$ ? with $t \rightarrow t / \xi=\rho$ and $\omega_{0} \rightarrow \omega_{0} \cdot \xi=v$. Applying this to the Morlet mother function leads to:

$$
\psi_{M}(t)=e^{j \omega_{0} t}\left(e^{-0.5 \rho^{2}}-\sqrt{2} e^{-0.25 v_{0}^{2}} e^{-\rho^{2}}\right) .
$$

The following condition is important for the reversibility of the WaveletTransform:

$$
C_{\psi}=\int_{-\infty}^{\infty} \sqrt{2 \pi \xi} e^{-0.25\left(\eta-\omega_{0}\right)^{2} \xi^{2}}\left(e^{-0.25\left(\eta-\omega_{0}\right)^{2} \xi^{2}}-e^{-0.25 v^{2}}\right) .1 /|\eta| d \eta<\infty
$$

with $\eta=2 \pi / T$ ( $\mathrm{T}=$ period).

Considering the scaling and shifting factors $a$ and $b$, as well as the WaveletTransform $X_{M}$

$$
X_{M}=\frac{1}{\sqrt{|a|}}\left\{\int_{-\infty}^{\infty}\left(X_{r} \cdot A_{1}+X_{i} \cdot A_{2}\right) \cdot d t-j \int_{-\infty}^{\infty}\left(X_{r} \cdot A_{2}-X_{i} \cdot A_{1}\right) \cdot d t\right\}
$$

Thus, with $\chi=(t-b) /(\xi a), B=\left(e^{-0.5 \chi^{2}}-\sqrt{2} e-\chi^{2} e^{-\left(\frac{\omega_{0} \xi}{2}\right)^{2}}\right), A_{1}=\cos \left(\omega_{0} \chi\right)$ and $A_{2}=B \sin \left(\omega_{0} \chi\right)$ the energy density can be defined as 


$$
\begin{gathered}
E=\left|X_{M}(b, a)\right|^{2}=\text { Energy density. } \\
E_{T}=\frac{1}{C_{\psi}} \int_{-\infty}^{\infty} \int_{-\infty}^{\infty}\left|X_{M}(b, a)\right|^{2} \frac{d b \cdot d a}{a^{2}}=\text { Total energy density. }
\end{gathered}
$$

The time-frequency window is defined as

$$
\left[a M_{\Psi t}+b-a \Delta \Psi_{t}, a M_{\psi t}+b+a \Delta \psi_{t}\right] \times\left[\frac{M_{\Psi \eta}}{a}-\frac{\Delta \Psi_{e} t a}{a}, \frac{M_{\Psi \eta}}{a}+\frac{\Delta \Psi_{\eta}}{a}\right]
$$

\section{References}

Arfa-Kaboodvand K., Groten E., 1998, Einsatz des Wavelet-Transforms zur Untersuchung der kurzperiodischen Variation der Polbewegung. Zeitschrift für Vermessungswesen, 123, 8, 259-265.

Arfa-Kaboodvand K., Groten E., Zavoti J., Varga P., 1999, Stochastische und deterministische Analyse sowie Modellierung der Erdrotation mit Schwerepunkt: Polschwankung und $\Delta$ UT1 (LOD), basierend auf GPS-Daten (3. DFG-Rundgespräch zum Thema Bezugssysteme), Mitteilungen des Bundesamtes für Kartographie und Geodäsie, Verlag des Bundesamtes für Kartographie und Geodäsie, 5, 126-133.

Arfa-Kaboodvand K., Groten E., 1999, In subdiurnal effects in Earth rotation, Studia geophysica et geodaetica, Academy of sciences of the Czech Republic, 3 (43), 275-283.

Chao B.F., Ray R. D., Gipson J. M., Egbert G. D., and Ma C., 1996, Diurnal/semidiurnal polar motion excited by oceanic tidal angular momentum, Journal of Geophysical Research, Vol. 101, No. B9, 20,15120,163 .

Barnes R. T. H., Hide R., White A. A. and Wilson C. A., 1983, Atmospheric angular momentum fluctuations, length-of-day changes and polar motion, Proc. Roy. Soc. London, Ser. A, 387, 31-73.

Brosche P., Seiler U., Sündermann J. and Wünsch J., 1989, Periodic Changes in Earth's Rotation Due to Oceanic Tides, Astronomy and Astrophysics, 220, 318-320.

Freedman A.P., Steppe J.A., Dickey J.O., Eubanks T.M., and Sung L.-Y., 1994, The short-term prediction of univesal time and length of day using atmospheric angular momentum, J. Geophys. Res., 99, 6981-6996.

Lichten S.M., Marcus S.L. and Dickey J.O., 1992, Sub-Daily resolution of earth rotation variations with global positioning system measurements, Geophysical Research Letters, 19 (6), 537-540.

Munk W. H. and McDonald G. J. F., 1960, The rotation of the Earth, Cambridge University Press, New York.

Popiński W. and Kosek W., 1994, Wavelet transforms and its application for short period earth rotation analysis. Planetary Geodesy, Nr. 22, Vol. 29, 75-86. 
Ray R.D., Steinberg D.J., Chao B.F. and Cartwright D.E., 1994, Diurnal and semidiurnal variations in the Earth's rotation rate included by oceanic tides, Science, 264, 830-832.

Rothacher M., 1998, Recent Contributions of GPS to Earth Rotation and Reference Frames, Habilitationsschrift, Druckerei der Universität Bern.

Seiler U., 1991, Prediction changes of the angular momentum budget due to the tides of the world ocean, Journal of Geophysical Research, 96 (B6), $287-300$.

Zharov V. E., Gambis D., 1996, Atmosperic tides and rotation of the Earth., Journal of Geodesy, 70, 321-326. 